\title{
IDENTIFICATION OF FACTORS IN THE DISPERSION OF VOLATILE SUBSTANCES OF THE INTERNAL ENVIRONMENT OF A CHEMICAL UNIVERSITY LABORATORY
}

doi:10.1136/injuryprev-2012-040590m.25

0 Peña, L Preciado, S Franco, M Medina, F Ramírez, C Mata. University of Guadalajara, Jalisco, Mexico

Background Volatile chemicals (VOCs) affect people's airways and maybe to be a risk factor to health if there is little culture of prevention, management and regulation in budding interior environments.

Objective To identify factors that may increase the concentration of VOCs in the indoor environment of a university chemistry laboratory during student practice.

Method The concentration was measured by portable flame ionisation detector in units of parts per million ( $\mathrm{ppm}$ ). It recorded three conditions: (a) with three extractors in to on and unlit, (b) practices study with volatile substances and unused, (c) instead of monitoring: front and back of the lab.

Results The variability of VOCs ppm for each of the effects, statistically significant difference test, comparing their mean square against an estimate of experimental error. In this case, two effects have $p<0.05$, indicating that they are significantly different from zero with a confidence level of $95.0 \%$. When exhaust fans are running, it is reported that $1578 \mathrm{ppm}$ smaller than amount when not in operation (1804 ppm). The average concentration of VOCs is reported of $2.03 \mathrm{ppm}$ without using volatile substances and 
increased to $28.27 \mathrm{ppm}$ when used, locating this concentration at the bottom of the laboratory.

Conclusions Contribution The concentration of VOCs increases when exhaust fans are not working and dispersed when operating only two of three extractors. With three fans, the VOCs were concentrated in a space and may be risk to health. 